\title{
Interfaces
}

INTERFACES Image Texte Language

$36 \mid 2015$

Synaesthesia

\section{Retour à l'Élysée : le jardin anglais revisité}

\section{Antoine Hatzenberger}

\section{(2) OpenEdition}

Journals

Édition électronique

URL : http://journals.openedition.org/interfaces/246

DOI : 10.4000/interfaces. 246

ISSN : 2647-6754

Éditeur :

Université de Bourgogne, Université de Paris, College of the Holy Cross

Édition imprimée

Date de publication : 1 janvier 2015

Pagination : 305-318

ISBN : 9780983175247

ISSN : $1164-6225$

Référence électronique

Antoine Hatzenberger, «Retour à l'Élysée : le jardin anglais revisité », Interfaces [En ligne], 36 | 2015, mis en ligne le 01 janvier 2018, consulté le 05 janvier 2021. URL : http://journals.openedition.org/ interfaces/246 ; DOI : https://doi.org/10.4000/interfaces.246

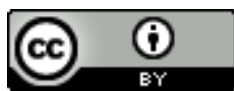

Les contenus de la revue Interfaces sont mis à disposition selon les termes de la Licence Creative Commons Attribution 4.0 International. 


\title{
RETOUR À L'ÉLYSÉE : LE JARDIN ANGLAIS REVISITÉ
}

\author{
Antoine Hatzenberger
}

«In all, let Nature never be forgot » Alexander Pope, Epistle to Lord Burlington

Retour à l'Élysée, le verger de Julie dans La Nouvelle Héloïse de Jean-Jacques Rousseau, parce que ce jardin est bien connu et a déjà été parcouru en tout sens ; retour aussi, parce que la visite de ce jardin offre, à chaque détour, des perspectives différentes. Entre les nombreuses approches possibles, ce jardin peut être intégré à une étude d'ensemble des modèles politiques dans l'œuvre de Rousseau, ou abordé du point de vue de l'histoire du genre de l'utopie (Hatzenberger 2010, 2012). Nouveau retour à l'Élysée donc, ici, du point de vue de l'esthétique des jardins. En effet, considérée en tant que description, indépendamment de son rôle narratif dans l'architecture du roman, ou de sa place dans l'ensemble de l'œuvre de Rousseau, la lettre 11 du quatrième livre de La Nouvelle Hélö̈se (1761) représente un grand intérêt pour l'histoire de la réception du style horticole venu d'Angleterre. L'Élysée offre en effet un très bel exemple de croisement des influences et d'hybridité stylistique, puisque les références critiques y sont nombreuses : jardins français, chinois et anglais. Et sur tous ces points, les travaux de Michel Baridon sur l'histoire des jardins sont indispensables pour se guider dans la lecture du jardin de Julie ${ }^{1}$.

\section{L'histoire des jardins de Michel Baridon}

Dans son article « Jardins et paysage : existe-t-il un style anglais ?» (1986), Michel Baridon répondait positivement à cette question, et préconisait de s'en tenir à la dénomination " jardin anglais » (plutôt que " pittoresque » ou " anglo-chinois ») pour désigner le jardin irrégulier dont la mode avait été lancée en Angleterre dès le début des années 1730. Dans cet article, Baridon dégageait les

Cet article reprend une étude présentée lors de la session organisée par Laurent Châtel « La culture du jardin dans l'Europe des Lumières. In memoriam Michel Baridon » lors du $13^{\text {e }}$ Congrès international d'études du XVIII ${ }^{\mathrm{e}}$ siècle, à Graz (Autriche), le 28 juillet 2011. 
caractéristiques propres à cet « imaginaire du paysage » et à ce nouveau style paysager, ce qu'il a appelé le « jardin de la sensibilité » (443), en mettant en avant l' " irrégularité » de ce « style asymétrique et sinueux » (428-429).

Dans « L'imaginaire antique et le palladianisme des Lumières » (1995), Baridon est revenu sur ces définitions, à partir d'une lecture tout à la fois architecturale, littéraire, politique et épistémologique des grands modèles que sont Chiswick et Stowe. Construisant une histoire holistique des idées, en croisant les différentes composantes culturelles d'une époque, il mettait en résonance des analyses des jardins et des villas (en faisant la part des influences respectives du mythe antique et du mythe gothique), des analyses des modèles politiques dans l'Angleterre du XVIII siècle (notamment du système de constitution mixte), des analyses de l'esthétique de la sensibilité inspirée de Locke, et des analyses du style palladien en littérature. Dans cet article synthétique, Baridon mettait en évidence l'« organisation polycentrique » des jardins et le fait qu'alors « la campagne [se faisait] paysage pour envahir le jardin » (118). Il notait qu' « à Stowe, quand bien même de longues allées rectilignes sont longtemps demeurées, l'asymétrie s'installe délibérément avec la création des Elysean Fields, dont le programme politique est aussi moderne que les formes sinueuses et la liberté des eaux » (120).

Si on ajoute à ces deux articles les grands textes sur les jardins regroupés, commentés, parfois traduits, et rendus ainsi plus accessibles par Baridon dans son anthologie Les Jardins : paysagistes, jardiniers, poètes (1998), on constatera encore une fois toute l'importance des analyses et des références fournies par ses travaux pour revisiter l'Élysée de La Nouvelle Hélö̈se - où l'on trouve justement une référence explicite aux jardins de Stowe.

Mais c'est surtout l'article « Utopie et sensibilité : le jardin des Lumières » (1986) qui vient confirmer la nécessité d'élargir l'enquête sur les utopies des Lumières à la prise en considération des jardins, puisque cet article a posé les fondements historiques d'une problématique mettant en relation l'œuvre de Rousseau et le thème de l'utopie, par l'entremise du troisième pôle que constitue le jardin. Dans cet article magistral, Baridon opérait, d'une part, une conjonction entre l'analyse esthétique de la culture des jardins et l'histoire des idées politiques, et, d'autre part, une comparaison des jardins anglais et français. Fournissant la trame historique et les outils conceptuels indispensables aux analyses des descriptions de jardins dans La Nouvelle Héloïse de Rousseau, cet article nous convie ainsi à un retour à l’Elysée.

Dans l'article «Utopie et sensibilité », Baridon a esquissé l'histoire de l'imaginaire du paysage au XVIII ${ }^{\mathrm{e}}$ siècle, en réfléchissant aux différents modes de relation entre le jardin et l'utopie. Il opposait la période de la première génération des Lumières, représentée par Voltaire, accordant 
la primauté à la raison, et encore attachée à « la sobriété classique » (119), à une deuxième époque, celle du sentiment, dont Rousseau est l'un des représentants, la transition étant assurée par le « goût palladien » de Montesquieu (123). Baridon a montré comment le jardin a changé de statut symbolique, passant du statut de " refuge de ceux qui désespèrent de l'utopie » (117), lorsque Versailles incarnait l'utopie autoritaire faisant plier les hommes et la nature devant l'autorité royale, au statut d' " utopie passéiste » (126) ensuite, lorsque le jardin anglais s'était fait la figuration nostalgique de l'utopie du « républicanisme antique » (125) ou d'une « utopie sociale » comme à Stowe (134), au statut d' « utopie nouvelle » enfin, dont on trouvera le meilleur exemple à Ermenonville.

Baridon suggérait dans cet article que c'est « Rousseau [qui] rend au jardin une dimension utopique » (118), dans la mesure où il avait " conçu le jardin comme un lieu de régénération où l'Homme pouvait espérer reconquérir un jour sa pureté originelle » (130) - ce dont le marquis de Girardin avait su se souvenir. Et Baridon ajoutait : «Utopie peut-être, mais utopie qui n'aurait jamais vu le jour sans la naissance du parc paysager en Angleterre. » Comme en passant, de manière discrète mais récurrente, Baridon faisait donc référence à Rousseau dans son panorama de l'évolution de l'esthétique des jardins du XVIII ${ }^{e}$ siècle, encourageant ainsi des analyses locales consacrées spécifiquement au texte de Rousseau sur les jardins.

Certes, bien d'autres études existent sur ce point. Mentionnons en particulier l'article de Peter Conroy (1982) sur le jardin polémique chez J.-J. Rousseau, et l'ouvrage de James F. Jones (1978) qui inscrivait dans une séquence significative les lieux emblématiques de La Nouvelle Héloïse : les montagnes du Valais (I, 23), le domaine de Milord Edouard Bomston dans le Yorkshire (II, 3), les îles des mers du Sud (IV, 3), le domaine de Wolmar à Clarens (IV, 10) et, enfin, point culminant du roman, l'Élysée de Julie (IV, 11)². Mais il faut réserver une place à part à l'article de Michel Baridon, qui esquisse une histoire de l'art des jardins en dégageant le processus dynamique qui est précisément à l'œuvre dans la description du domaine de Clarens et de l'Élysée de Julie. Le schéma qu'il reconstitue fournit une grille de lecture particulièrement pertinente pour l'analyse des intertextualités paysagères dans La Nouvelle Héloïse. Le mouvement tel que l'analyse Baridon est schématiquement le suivant : à la remise en cause du modèle classique de Versailles par le jardin anglais succède la critique du jardin politique du « républicanisme aristocratique » (131) par le jardin naturel. Or, on retrouve précisément chacun de ces moments dans le domaine de Clarens, non plus chronologiquement, distincts dans le temps, mais inscrits dans l'espace, sous forme de strates ou de palimpsestes. Ce qui nous donne : 1) le jardin baroque du baron d'Étange ; 2) le nouveau jardin de Wolmar ; 3) le verger de Julie ; et à l'intérieur même de l'Élysée, un mélange de styles, évoqués sur un mode critique.

Voir aussi Marin 1992 ; Haquette 2001 ; Goubier-Robert 2003. 


\section{Rousseau et les jardins anglais}

La relecture des deux célèbres lettres de Saint-Preux dans La Nouvelle Héloïse au prisme des indications de Michel Baridon donne bien un aperçu de la richesse de la culture du jardin dans l'Europe des Lumières. Ces lettres contiennent tout à la fois des indices de l'entrelacement des influences, et des formulations d'une critique esthétique des différents styles de jardins, fournissant ainsi des éléments quant à la réception des jardins anglais sur le continent européen autour de 1760 .

Une approche biographique de la recherche des sources pourrait laisser supposer que Rousseau eût pu voir le domaine archétypal de Lord Burlington à Chiswick, passant par l'Ouest de Londres lors de son exil anglais en 1766-1767. En tout cas, il n'était pas passé bien loin de la villa palladienne, Chiswick House. En revanche, ce qui est sûr, c'est que si Rousseau mentionne Stowe l'Élysée de William Kent - de seconde main dans La Nouvelle Hélö̈se, il a en revanche fait part dans sa correspondance des effets que firent directement sur lui les paysages qu'il put découvrir dans les campagnes anglaises, témoignant ainsi de sa familiarité avec la culture du jardin dans l'Europe des Lumières.

Ainsi de sa description du domaine de Wootton Hall dans le Derbyshire, où il séjourna quelques temps, et dont il décrit dans une lettre les " promenades de plain-pied sur la plus belle pelouse de l'univers » et « des jardins à l'anglaise, moins peignés, mais de meilleur goût que ceux des Français ». Parlant du parc, il ajoutait cette précision : « si l'on peut donner ce nom à une enceinte à laquelle on a laissé toutes les beautés de la nature », saisissant bien par là l'un des traits de l'esprit du style paysager³.

Dans la première lettre qu'il écrivit, de Wootton, le 20 octobre 1766 (CC XXXI, lettre 5482), à la duchesse de Portland, compagne d'herborisation qui allait devenir l'une de ses correspondantes en matière de botanique (Cook 2007), Rousseau laissait poindre, outre son regain d'intérêt pour les plantes,

3 Lettre à Marianne-Françoise de Luze, à Wootton, 10 mai 1766, CC XXIX, lettre 5197 : «Figurez-vous, Madame, une maison seule, $[. .$.$] bâtie à mi-côte sur le penchant d'un vallon dont la pente est assez interrompue pour laisser$ des promenades de plain-pied sur la plus belle pelouse de l'univers. Au devant de la maison règne une grande terrasse d'où l'œil suit dans une demi-circonférence de quelques lieues un paysage formé de prairies, d'arbres, de fermes éparses, de maisons plus ornées, et bordée en forme de bassin par des coteaux élevés qui bornent agréablement la vue quand elle ne pourrait aller au-delà. [...] Au bout de la terrasse à gauche sont des bâtiments rustiques et le potager, à droite sont les bosquets et un jet d'eau. Derrière la maison est un pré entouré d'une lisière de bois laquelle, tournant au-delà du vallon couronne le parc, si l'on peut donner ce nom à une enceinte à laquelle on a laissé toutes les beautés de la nature. [...] aux environs on a le choix des promenades soit dans des prairies charmantes, soit dans des bois soit dans des jardins à l'anglaise, moins peignés, mais de meilleur goût que ceux des Français. » 
son intérêt pour les jardins qui les contiennent. Il lui écrit que « le jardin de [s]on excellent voisin, M. de Granville [le jardin de Calwich Abbey sur la rivière Dove], [lui] a donné le projet ambitieux d'en connaître les richesses ».

Dans une lettre du 17 avril $1772^{4}$, où il se souviendra aussi du jardin des Portland à Bulstrode, Rousseau mentionne « le livre des jardins anglais », c'est-à-dire sans doute le premier tome du poème de William Mason, The English Garden (1772), dont la duchesse lui avait envoyé un exemplaire, en même temps que des graines. Dans sa lettre de remerciement, Rousseau assurait sa correspondante qu'il « en [doit] aimer le sujet, ayant été le premier en terre ferme à célébrer et faire connaître ces mêmes jardins ». Cette conscience d'avoir été un médiateur se justifie en référence à La Nouvelle Héloïse et à certains éléments de sa description de l'Élysée de Julie. En effet, dans son roman épistolaire, Rousseau avait fait référence au " parc célèbre de Milord Cobham à Staw » dans le Buckinghamshire (484) et au « parc de St. James » (note, 483), et il avait aussi inséré dans une autre lettre un tableau du domaine de Milord Edouard dans le Yorkshire ${ }^{5}$, mêlant ainsi descriptions de jardins réels et de jardins imaginaires.

Il y a donc dans La Nouvelle Hélö̈se plusieurs modèles de jardins qui sont en rapport les uns avec les autres, faisant système, dessinant ensemble un discours sur le jardin. Rousseau marque les grandes époques de l'évolution des styles, en présentant la description de la métamorphose des jardins au XVIII ${ }^{\mathrm{e}}$ siècle, et en exposant les principes qui ont présidé à leurs transformations.

Le domaine du baron d'Étange était un jardin classique, symétrique, planté de haies d'ifs taillés en palissades et de rangées de tilleuls, et dont le parterre était orné d'un jet d'eau. Ce jardin d'origine, symbole de l'ancien style, Wolmar l'a transformé en faisant du parterre un grand potager, c'est-à-dire en substituant partout l'utile à l'agréable. Le conseil avait été formulé dans la lettre de Milord Edouard dans le deuxième livre : il faut ménager des perspectives, mais il faut aussi faire fructifier ses terres.

$4 \quad$ CC XXXIX, lettre 6934 : « Avez-vous pu douter un instant, Madame la Duchesse, que je n'eusse reçu avec autant d'empressement que de respect le livre des jardins anglais que vous avez bien voulu penser à m'envoyer? Quoique son plus grand prix fût venu pour moi de la main dont je l'aurais reçu, je n'ignore pas celui qu'il a par lui-même, puisqu'il est estimé et traduit dans ce pays, et d'ailleurs j'en dois aimer le sujet ayant été le premier en terre ferme à célébrer et faire connaître ces mêmes jardins. Mais celui de Bulstroode [Bulstrode] où toutes les richesses de la nature sont rassemblées et assorties avec autant de savoir que de goût mériterait bien un chantre particulier. »"

5 La Nouvelle Héloïse, II, 3, 199 : « J'ai dans le Duché d'York une terre assez considérable, qui fut longtemps le séjour de mes ancêtres. Le château est ancien, mais bon et commode ; les environs sont solitaires, mais agréables et variés. La rivière d'Ouse qui passe au bout du parc offre à la fois une perspective charmante à la vue et un débouché facile aux denrées ». 
Un domaine doit à la fois plaire et rapporter (II, 3). Or, ces principes de rationalisation économique des jardins avaient été exposés par Joseph Addison dans Le Spectateur en $1712^{6}$. C'est lui qui, dans des termes très ressemblants à ceux que l'on trouvera dans La Nouvelle Héloïse, imaginait de faire « d'un domaine entier une espèce de jardin par de fréquentes plantations, qui tourneraient aussi bien au profit qu'au divertissement du propriétaire $\gg{ }^{7}$. Michel Baridon, qui a inclus cet article d'Addison dans son anthologie, commente ainsi cette transformation des parcs en "status-symbols", impressionnants par l'étendue mais pas trop coûteux d'entretien » : « En finir avec les parterres de broderie, renoncer aux jets d'eaux, laisser serpenter les rivières sans creuser de bassins, confier aux moutons l'entretien des pelouses, tout cela c'était rendre la liberté rentable » (Baridon 1986, 435).

\section{Critique des jardins}

À cette dimension descriptive des lettres de La Nouvelle Héloïse se superpose une dimension réflexive et critique. En effet, Rousseau va opérer une critique systématique des différents types de jardins, dont il accentue parfois certains traits caractéristiques jusqu'à la caricature. Il s'agit bien d'un discours sur les jardins, un discours argumenté et illustré par de nombreux exemples.

Première critique, celle des jardins ornementaux, qui s'éloignent trop de « ce qui se trouve dans la campagne » naturellement. Cette critique de l'artifice touche en même temps le jardin classique et le jardin paysager, indifféremment donc styles français et anglais, puisque Rousseau vise le jardin qu' « un homme riche de Paris ou de Londres » aurait commandé à un architecte (480). La critique porte sur la symétrie des alignements et des allées, sur la géométrisation des parterres et des arbres taillés, et sur la débauche de l'ornementation (figures, vases ou statuettes). Dans toute la lettre, sont critiquées les lignes droites, les étoiles - tout ce qui est tracé avec " l'équerre et la règle » (482). Là aussi, l'argumentation et les exemples rappellent quelque chose : en l'occurrence le catalogue parodique des extravagances de l'art topiaire que l'on trouve dans l'article de Pope de 1713, également repris dans l'anthologie de Baridon'.

6 Le jeune Rousseau lisait Le Spectateur (traduit en français dès 1714) chez Mme de Warens (Confessions, III, $O C \mathrm{I}, 110-111)$.

7 The Spectator, $\mathrm{n}^{\circ}$ 414, 25 juin 1712 ; cité dans Baridon 1998, 843

$8 \quad$ The Guardian, $\mathrm{n}^{\circ} 173,1713$; Baridon 1998, 846. 
La seconde critique porte sur les jardins botaniques. Rousseau moque le « goût ridicule [...] de ces petits curieux, de ces petits fleuristes qui se pâment à l'aspect d'une renoncule, et se prosternent devant des tulipes » (481). Il illustre ce point en décrivant la visite d'une exposition de fleurs précieuses à Londres, où brillaient " pompeusement tous les trésors de la Hollande sur quatre couches de fumier ». À la critique du luxe s'ajoute un argument récurrent chez Rousseau : les plantes doivent être contemplées dans leur milieu naturel. De ce fait, Rousseau désapprouve à la fois les jardins de curieux et les jardins d'apothicaires. On retrouve cette critique constante des Confessions à la septième promenade des Rêveries du promeneur solitaire, en passant par les lettres sur la botanique : Rousseau dissocie le goût authentique pour l'herborisation de l'intérêt que l'on peut prendre aux jardins de plantes précieuses ou médicinales. Cet argument se double d'une critique des plantes exotiques et des cultures hors-sol, à laquelle Rousseau préfère toujours les fleurs autochtones, contemplées de manière désintéressée dans leur milieu naturel.

Troisièmement, la critique porte sur le jardin chinois, brièvement évoqué. Ce qui est visé dans les « jardins de la Chine », outre leur coût, dû en particulier à l'hyper-sophistication des installations hydrauliques, c'est le caractère trop artificiel et la profusion de l'ensemble.

Enfin, et cette critique est liée à la précédente, si aux roches, aux grottes et aux cascades artificielles des jardins chinois s'ajoutent à Stowe les fabriques (ruines, temples et anciens édifices), la critique se formule dans les mêmes termes à l'encontre de l'aspect hétéroclite de l'assemblage ainsi que des dépenses et du travail requis par de telles réalisations.

\section{L'anti-jardin}

En opposition à ces différentes formules, la solution que représente l'Élysée à l'équation complexe entre l'art et la nature peut être interprétée au moins de deux façons. En un sens, on pourrait dire que l'Élysée n'existe pas : ce serait une fiction théorique, un anti-modèle qui se construit dans le creux des critiques adressées à d'autres modèles, anciens ou à la mode du temps. Mais on pourrait tout aussi bien dire que l'Élysée est ce qui reste au terme du travail critique : ce serait le résultat abstrait d'une série de soustractions. Si l'Élysée emprunte certaines caractéristiques à chacun des modèles de jardins évoqués, il faut souligner qu'il s'agit d'une synthèse épurée de tous leurs défauts respectifs.

Ici l'on n'a transporté $n i$ terres $n i$ pierres, on n'a fait $n i$ pompes $n i$ réservoirs, on n'a besoin $n i$ de serres, $n i$ de fourneaux, $n i$ de cloches, $n i$ de paillassons. Un terrain presque uni a reçu des ornements très simples. Des herbes communes, des arbrisseaux communs, quelques filets 
d'eau coulant sans apprêts, sans contrainte, ont suffi pour l'embellir. C'est un jeu sans effort, dont la facilité donne au spectateur un nouveau plaisir. (484)

Dans l'Élysée, que cette description présente comme un jardin négatif(sans rien) et minimaliste (simple, commun, facile), pas d'alignements, mais des « allées tortueuses et irrégulières » (472), recouvertes de mousse, selon un secret envoyé d'Angleterre ; pas de jet d'eau, de bassin ou de cascade, mais un ruisseau ; pas de « petits bosquets à la mode », mais des gazons, des fleurs des champs et des plantes aromatiques ; pas d' « ifs taillés en dragons », mais des oiseaux en liberté. Partout, le principe directeur est que l'art doit être discret et ne doit pas masquer ou gâcher la nature. Les éléments doivent être simples, ainsi que leur agencement.

Il faudrait ajouter encore - ultime critique - que tout ce dispositif entre en contradiction sur bien des points avec le domaine de Wolmar : féminin contre masculin, loisir contre économie, plaisir contre devoir, nature contre travail. Ce changement de paradigme, qui peut se résumer en une dialectique de l'utile et de l'agréable, constitue une critique politique interne à l'économie rustique de la country house. L'Élysée de Julie serait un contre-point au domaine de Clarens (Hatzenberger 2010).

Mais pour en rester ici à l'esthétique des jardins, il faut rappeler, au terme de ce détour par l'Élysée, ce qui se dit, dans La Nouvelle Hélö̈se, de la culture du jardin dans l'Europe des Lumières, tant ce texte semble offrir un témoignage sur les influences croisées dans l'esthétique des jardins du XVIII ${ }^{e}$ siècle, sur l'histoire de la réception des jardins et de la diffusion des nouveaux styles.

\section{Un jardin paradoxal}

La lettre de La Nouvelle Héloïse (IV, 11), que tout porte à lire comme un véritable essai sur les jardins, apporte un éclairage sur la réception du jardin oriental en Europe, avec la référence « aux jardins de la Chine ». Surtout, elle met en évidence toute l'ambiguïté de la référence au jardin anglais. En effet, cette référence est contrastée. D'un côté, Rousseau démontre la connaissance qui était la sienne de la nouvelle esthétique du jardin, et de toute la grammaire horticole de la topiaire et des fabriques, par des allusions élogieuses aux jardins anglais, notamment dans sa correspondance, et par des manifestations concrètes de cette admiration dans sa magnifique description de l'Élysée. Mais, d'un autre côté, aussi, Rousseau critique le jardin de l' « homme riche de Londres »; il ridiculise une visite dans un «flower show»; et il formule une critique des réalisations de Kent et de Brown à Stowe, à partir de la description qu'il avait pu en lire9. On est donc bien renvoyé à une série de paradoxes.

9 Dans l'Épître à Lord Burlington de Pope, ou dans le Mercure de France de février 1750 ; voir OC II, 484, note 2. 
Premier paradoxe : le discours de Rousseau sur les jardins est à la fois élogieux et critique. Certes, il critique par ailleurs le théâtre et les romans, tout en écrivant des pièces et La Nouvelle Héloise... Mais dans le cas des jardins, le paradoxe n'est pas thématisé comme il l'est pour les autres formes d'art ( $c f$. préface à Narcisse). Ce qu'on peut remarquer dans ce cas précis, c'est que Rousseau ne fait pas de jardins : il se contente de les apprécier, de les décrire et de les imaginer, mais toujours en référence à une nature originaire.

Ce point est lié à un paradoxe touchant aux principes fondamentaux de la philosophie de Rousseau : l'apologue de la nature s'extasie devant un jardin, c'est-à-dire un morceau de nature aménagé par les hommes. Comment concilier la référence à l'état de nature (Saint-Preux compare l'Élysée à des îles vierges) et la description de jardins, qui restent bien toujours - malgré toutes les apparences entretenues - des espaces cultivés ? On le sait, nul retour en arrière n'est vraiment possible. Aussi naturel qu'il puisse paraître, le jardin ne fait jamais que renvoyer à une image plus ou moins convaincante de la nature. Alors que le domaine de Wolmar représente la culture (le travail), l'Élysée est une allégorie de la nature, une parenthèse, une utopie, un détour, l'invitation à un impossible retour.

Tout se passe finalement comme si Rousseau ménageait des distances par rapport à son objet afin de pouvoir véritablement constituer une esthétique - une esthétique du paysage qui reste fondamentalement indexée à une philosophie morale et à une conception de l'économie politique, gardant bien le détracteur des artifices, du luxe et de l'ostentation, de vouloir s'identifier à un propriétaire de jardin.

Autre paradoxe : la place de Rousseau dans l'histoire des jardins est à la fois centrale et marginale. Centrale, parce qu'il agit comme un passeur. L'Élysée reflète en effet l'esprit du style nouveau, et sert à son tour de modèle pour de nouvelles créations paysagères. La Nouvelle Héloïse est au cœur de la nouvelle esthétique, et le jardin de Julie est devenu un modèle. Marginale, pourtant, parce que Rousseau est finalement passé à côté des grands domaines paysagers les plus emblématiques de son temps (notamment Chiswick), comme il avait aussi sans doute dû renoncer à la lecture de The English Garden, dont la duchesse de Portland lui avait envoyé un exemplaire en anglais ${ }^{10}$. Une note de

10 Lettre à la duchesse de Portland, 19 mai 1772, CC XXXIX, lettre 6940 : « Je dois, Madame la Duchesse, le principal plaisir que m'ait fait le Poème sur les Jardins Anglais que vous avez eu la bonté de m'envoyer, à la main dont il me vient. Car mon ignorance dans la langue anglaise qui m'empêche d'en entendre la poésie ne me laisse pas partager le plaisir que l'on prend à la lire. Je croyais avoir eu l'honneur de vous marquer, Madame, que nous avons cet ouvrage traduit ici, vous avez supposé que je préférais l'original et cela serait très vrai si j'étais en état de le lire ; mais je n'en comprends tout au plus que les notes qui ne sont pas à ce qu'il me semble la partie la plus intéressante de l'ouvrage. Si mon étourderie m'a fait oublier mon incapacité $\mathrm{j}$ 'en suis puni par mes vains efforts pour la surmonter. Ce qui n'empêche pas que cet envoi ne me soit précieux comme un nouveau témoignage de vos bontés et une nouvelle marque de votre souvenir. " 
R. A. Leigh indique de toute façon que Rousseau confondait le poème de Mason avec la traduction des Observations on Modern Gardening de Thomas Whately, parues en 1770 et traduites en 1771 sous le titre de L'Art de former les jardins modernes, ou l'art des jardins anglais (CC XXXIX, 43). En ce sens, on pourrait dire que Rousseau est passé à côté du jardin anglais.

Autant d'occasions manquées - ou de distances délibérément maintenues - qui rendent difficile l'établissement exact des sources et des datations, et qui poussent à avancer l'hypothèse selon laquelle il faut peut-être s'en émanciper pour considérer le jardin rousseauiste à part entière comme concomitant, ou concurrent, au jardin anglais, et non plus comme un style dérivé. Il y aurait ainsi véritablement un jardin rousseauiste sui generis.

\section{Le jardin de Rousseau}

Pour cerner ce qu'on appellera l'esthétique du paysage-jardin propre à Rousseau il faut considérer les points communs de l'Élysée avec d'autres lieux, en particulier dans les Rêveries du promeneur solitaire (1778), œuvre dont les descriptions de paysage (Eigeldinger 1973, Tripet 1992) l'île de Saint-Pierre, les Alpes - participent de la même esthétique que celle qui s'affirme dans le jardin de Julie.

En s'ouvrant sur le spectacle des « rives romantiques » (1040) et en se concluant sur celui des « romanesques rivages » $(1048)^{11}$, la Cinquième promenade requalifiait la perception de la nature en fonction de catégories esthétiques nouvelles qu'elle contribuait à établir. Les éléments saillants du paysage sont la " verdure », les « prairies », les " asiles ombragés de bocage », les « contrastes », les « accidents », mais aussi le chant des oiseaux et le bruit de l'eau $(\mathrm{V}, \S 2)$. Comme dans l'Élysée, la perception esthétique du paysage est une expérience synesthétique. Dans la Septième promenade, on retrouve cette même harmonie des éléments : " brillantes fleurs, émail des prés, ombrages frais, ruisseaux, bosquets, verdure » (§21). L'équation rousseauiste est la suivante : « spectacle de la nature $»=$ fleurs + eau vive + oiseaux.

Dans la Septième promenade se mêlent le modèle classique de la pastorale, le modèle pictural du paysage composite et le modèle du jardin paysager. Ce point de vue esthétique sur la nature

11 Selon René-Louis de Girardin, romanesque « désigne la fable du roman », alors que le mot anglais romantique « désigne la situation et l'impression touchante que nous en recevons » (De la composition des paysages [1777], 99 , note a). 
paysagée marque un moment remarquable dans l'histoire du regard et des typologies stylistiques. Le " refuge ignoré de tout l'univers » que Rousseau trouve dans les " anfractuosités de la montagne » (1070-1071) correspond à un topos récurrent dans la description du paysage de la Meillerie dans $L a$ Nouvelle Héloïse (« réduit sauvage et désert, mais plein de ces beautés qui ne plaisent qu'aux âmes sensibles »), ou dans la forêt de Montmorency (Confessions). De telles descriptions relèvent pour une part du bosquet, c'est-à-dire de la forêt idéalisée aux essences mélangées, et du locus amœnus tel que Ernst Robert Curtius en a dégagé les invariants : dans ce paysage idéal, ce lieu est « une "tranche" de nature belle et ombragée ; son décor minimum se compose d'un arbre (ou de plusieurs), d'une prairie et d'une source, ou d'un ruisseau. À cela peuvent s'ajouter le chant des oiseaux et des fleurs. Le comble sera atteint, si l'on y fait intervenir la brise $»^{12}$. « [D]e la verdure et des fleurs, du ciel azuré, des bergers amoureux et des laboureurs robustes » $(\S 18)$ : c'est le paysage de la pastorale.

La Cinquième promenade souligne la beauté des « contrastes » (1040) du « superbe et ravissant coup d'œil du lac et de ses rivages » (1045), d'une petite île « variée dans ses terrains et dans ses aspects » (1041). La Septième promenade renforce encore cette esthétique picturale des tableaux composés en célébrant le spectacle du "mélange de la nature sauvage et de l'industrie humaine » (§ 27). Ces variantes de «mélange bizarre » (dont parle Rousseau dans une lettre du 20 janvier 1763) sont, selon Joachim Ritter, le propre de la vision esthétique du "paysage composite, qui fait se côtoyer sur un même espace représenté des lieux éloignés ou des références disparates » (Reichler 2002). La description du Haut-Valais dans La Nouvelle Hélö̈se relève de ce même schéma du « spectacle inattendu » donnant simultanément à voir différents aspects de la nature - « au levant les fleurs du printemps, au midi les fruits de l'automne » $(\mathrm{I}, 23,77)$. En comparant la source de la Reuse, la rivière du Val-de-Travers, avec la fontaine de Vaucluse dans une lettre célébrant la « variété des sites et des points de vue $»^{13}$, Rousseau invite à compléter la comparaison entre cette esthétisation du paysage alpestre avec la découverte du paysage par Pétrarque lors de l'ascension du Mont Ventoux (Hatzenberger 2013). Selon Joachim Ritter, cette nouvelle façon de voir marquait la naissance d'un intérêt désintéressé pour la nature en tant que paysage, c'est-à-dire, « la nature esthétiquement présente, se montrant à un être qui la contemple en éprouvant des sentiments » (Ritter 1997, 59). La campagne et la montagne ne deviennent paysages « que pour l'homme qui se tourne vers eux pour jouir librement de leur spectacle et pour être lui-même au sein de la nature, sans poursuivre de finalités pratiques ». La nature devient objet esthétique par le regard départi de tout intérêt direct, ou « matériel » comme le dit Rousseau

12 Curtius [1948] 1991 ; chap. X, « Le paysage idéal », 317.

13 Lettre au Maréchal de Luxembourg, n² 2457, 28 janvier 1763, CC XV, 113 et 115. 
(VII, § 15). C'est cette nouvelle esthétique qui explique, a contrario, la critique rousseauiste de la « botanique de cabinet et de jardin » (§23), dans les Rêveries, et déjà dans Julie.

La critique de Stowe et des jardins chinois visait le mode de l'assemblage du divers. Or, dans sa description de paysages, Rousseau donne la solution de l'harmonie d'un ensemble : le paysagejardin doit produire une synthèse parfaite des éléments qu'il comporte et des parties qui le composent. Comme l'a souligné Georg Simmel, dans sa « Philosophie du paysage », le paysage doit former « audelà des éléments, un nouvel ensemble, une nouvelle unité » (Simmel 1988, 231). C'est justement ainsi que fonctionne la référence au modèle des « jardins anglais » dans la description du paysage composite de la Suisse (VII, § 27), à la fois mélange dans l'unité et illimité dans des limites.

Le jardin rousseauiste serait donc une référence importance en lui-même, un parangon, et non pas un simple réceptacle du jardin anglais. Même, ce modèle esthétique a pu agir à rebours, en informant le paysage anglais et le regard que l'on portait sur lui. Ainsi, Girardin cherchera à reproduire l'Élysée à Ermenonville, après avoir cherché à retrouver l'Élysée aux Leasowes, selon le procédé de l'artialisation de la nature (Roger 1997). Un autre ami de Rousseau, l'Anglais George Simon Harcourt, avait lui aussi été influencé par La Nouvelle Hélö̈se (Voisine 1956, 35-37). Le jardin de NunehamCourtenay, près d'Oxford, avait été conçu par William Mason comme un hommage à l'auteur de Julie, et c'était d'ailleurs précisément une citation inspirée d'une note de la lettre sur l'Élysée qui ornait le portique donnant accès au jardin floral : "Si l'auteur de la Nature est grand dans les grandes choses, il est très grand dans les petites $»^{14}$. Selon Brooke Boothby, Rousseau avait sans doute été informé des marques de sa présence dans l'Élysée anglaise ${ }^{15}$. Ainsi, conscient de son rôle de pionnier dans la diffusion du jardin anglais sur le continent (comme il l'avait confié à la duchesse de Portland), il devait être également conscient de l'influence qu'exerçait son esthétique sur les paysages de l'île.

Rousseau participait ainsi, aux côtés notamment de Claude-Henri Watelet, avec lequel il était en contact ${ }^{16}$, et avec René-Louis de Girardin, à la constitution d'un style continental de jardin moderne.

$14 C f$. note, $O C$ II, 482. Le croisement des influences est plus accentué encore par la présence d'images rousseauistes dans le château du domaine de Nuneham, où le visiteur pouvait « contempler dans le vestibule deux vues en couleurs, l'une de la maison de Jean-Jacques à Motiers-Travers, l'autre de la tombe dans l'île des Peupliers ; cette dernière offerte par Girardin » (Voisine 1956, 36-37) - deux paysages rousseauistes.

15 Lettre de Brooke Boothby à George Simon Harcourt, 14 décembre 1777, CC XL, lettre 7145 : « When I saw Rousseau last I mentioned to him the circumstance of your lordship having placed his bust in your Elisée, \& something of the inscriptions ».

16 Dans une lettre à Rousseau du 10 février 1767, Harcourt évoque sa visite à Moulin-Joli, le jardin pittoresque de Watelet (CC XXXII, lettre 5724). 
L'Essai sur les jardins, De la composition des paysages, comme les Rêveries, et comme La Nouvelle Héloïse, mettaient en avant le modèle de la "ferme ornée » et du parc "romanesque ». Rousseau diffusait une esthétique du spectacle de la nature, dont l'influence allait être sensible chez les lecteurs anglais de La Nouvelle Hélö̈se, comme par exemple chez un Richard Hurd, qui témoignait ainsi de son admiration à William Mason : «But the rocks of Meillery \& the domestic transactions of Clarens are above everything that is extant in the world, of that kind. ${ }^{17} \gg$ Et comme le notera Nietzsche, peu amène pourtant par ailleurs envers lui : « [d]epuis Rousseau, l'on a découvert le sens de la beauté des sites alpestres et du désert » (Aurore, V, 427).

En définitive, tout ceci montre qu'en dépit de la position ambivalente ou paradoxale de Rousseau à l'égard du jardin, il n'est pas exclu de trouver une certaine cohérence à son approche. Il faut en effet remarquer que, de manière générale, toutes les critiques de Rousseau envers les jardins convergent vers une série d'oppositions, qui peuvent se résumer en la recherche d'un point d'équilibre entre le naturel et l'artificiel. Si Rousseau critique les grands travaux des architectes de jardins et le goût du luxe des propriétaires de domaines, c'est, comme ailleurs dans son œuvre, toujours au nom de la simplicité et de la sensibilité.

Malgré tout, il reste donc indéniable que Rousseau laisse transparaître dans plusieurs lieux importants de son œuvre un goût pour les jardins, un goût surtout théorique en relation avec sa philosophie de la nature et en marge de sa vraie passion pour l'herborisation. Dans le jardin de Julie, Saint-Preux aime à reconnaître la nature, de la même façon qu'à d'autres moments, le promeneur solitaire se plaira à trouver dans la nature des airs de jardins cultivés. Tout se joue finalement dans ce chiasme de la relation entre l'art et la nature. Et c'est de ces jeux de ressemblance, de ces analogies, qui accompagnent toutes les promenades possibles dans la nature naturelle ou dans la nature cultivée, que se nourrit le plaisir de l'imagination.

Autant de raisons, donc, pour faire retour à l'Élysée...

17 Lettre de Richard Hurd à William Mason, 30 mars 1761, CC VIII, appendice 239, « Quelques admirateurs de $L a$ Nouvelle Hélö̈se dans le clergé anglican ». 


\section{Ouvrages Cités}

BARIDON, Michel. «Utopie et sensibilité : le jardin des Lumières ». Les Lumières. 1986. Éd. Norbert Jonard. Vol. 2. Les Lumières : utopie et sensibilité. Dijon : Université de Bourgogne. 117-136.

— « Jardins et paysage : existe-t-il un style anglais ?». Dix-huitième siècle 18 (1986) : 427-446.

— «L'imaginaire antique et le palladianisme des Lumières ». Dix-huitième siècle 27 (1995) : 109-127.

— Les Jardins : paysagistes, jardiniers, poètes. Paris : Robert Laffont, 1998.

CONROY, Peter V. « Le jardin polémique chez J.-J. Rousseau ». Cahiers de l'association internationale des études françaises 34 (1982) : 91-105.

COOK, Alexandra. « Botanical exchanges: Jean-Jacques Rousseau and the Duchess of Portland ». History of European Ideas 33 (2007) : 142-156.

CURTIUS, Ernst Robert. La Littérature européenne et le Moyen Âge latin. 1948. Trad. J. Bréjoux [1956]. Paris : Presses Pocket, 1991.

EIGELDINGER, Marc. «Rousseau et le paysage végétal ». Poésie et métamorphoses. Neuchâtel : La Baconnière, 1973. GIRARDIN, René-Louis de. De la composition des paysages. 1777. Paris : Champ Vallon, 1993.

GOUBIER-ROBERT, Geneviève. «L'Élysée : leçon de morale dans un parc ». Jean-Jacques Rousseau et les arts visuels. Éd. Frédéric S. Eigeldinger. Annales de la Société J.-J. Rousseau 45 (2003) : 113-129.

HAQUETTE, Jean-Louis. « Le jardin de Julie dans La Nouvelle Héloïse : un état des lieux critiques ». Le Paysage : état des lieux. 2001. Éd. Françoise Chenet, Michel Collot et Baldine Saint-Girons. Bruxelles : Ousia. 175-194.

HATZENBERGER, Antoine. «La double utopie de Clarens : l'utile et l'agréable dans les jardins de Rousseau ». Utopies des Lumières. 2010. Éd. Antoine Hatzenberger. Lyon : ÉNS Éditions. 109-139.

— Rousseau et l'utopie : de l'état insulaire aux cosmotopies. Paris : Honoré Champion, 2012. 272-299.

— «Paysages de montagne : Pétrarque et Rousseau géographes ». Des Paysages. 2013. Éd. Mongi Bourgou et Antoine Hatzenberger. Manouba / Tunis : Centre de Publication Universitaire / École Normale Supérieure de Tunis. 171-180.

JONES, James Fleming. La Nouvelle Héloïse : Rousseau and Utopia. Genève : Droz, 1978.

MARIN, Louis. « Le jardin de Julie ». Lectures traversières. Paris : Albin Michel, 1992. 63-87.

REICHLER, Claude. La Découverte des Alpes et la question du paysage. Genève : Georg, 2002. 172-173.

RITTER, Joachim. «Le Paysage : fonction de l'esthétique dans la société moderne ». Trad. G. Raulet [Argile 16. 1978]. Besançon : Éditions de l'Imprimeur, 1997.

ROGER, Alain. Court traité du paysage. Paris : Gallimard, 1997.

ROUSSEAU, Jean-Jacques. Euvres complètes. 1959-1995. Éd. Bernard Gagnebin et Marcel Raymond. Paris : Gallimard, « Pléiade ».

—Correspondance complète. 1965-1998. Éd. R. A. Leigh. Oxford : Voltaire Foundation.

SIMMEL, Georg. « Philosophie du paysage ». 1913. La Tragédie de la culture. Trad. S. Cornille et Ph. Ivernel. Paris : Rivages, 1988.

TRIPET, Arnaud. « Rousseau et l'esthétique du paysage ». Annales de la Société J.-J. Rousseau 40 (1992) : 65-81. VOISINE, Jacques. J.-J. Rousseau en Angleterre à l'époque romantique. Paris : Didier, 1956. 